\title{
CONVEXITY AND CONCAVITY OF THE PERRON ROOT AND VECTOR OF LESLIE MATRICES WITH APPLICATIONS TO A POPULATION MODEL
}

\author{
Stephen J. Kirkland \\ Institute for Mathematics and its Applications \\ University of Minnesota \\ Minneapolis, Minnesota 55455 \\ Michael Neumann * \\ Department of Mathematics \\ University of Connecticut \\ Storrs, Connecticut 06269-3009
}

*Research supported by NSF Grants Nos. DMS-8901860 and DMS-9007030. 


\begin{abstract}
This paper considers the Leslie model of population growth and analyzes its asymptotic growth rate and its asymptotically stable age distribution as functions of the fecundity and survival rates of each age group in the population. This analysis is performed by computing first and second order partial derivatives of the Perron root and vector of a Leslie matrix with respect to each relevant entry in the matrix, with emphasis on the second partial derivatives. We discuss the signs of these derivatives as well as the qualitative implications that our results have for the Leslie model. Where possible, quantitative interpretations of the results are also given. Throughout, the techniques employ ideas from the theory of group generalized inverses.
\end{abstract}




\section{INTRODUCTION}

The purpose of this paper is to investigate first and second order effects of changes in fecundity and survival rates of various age groups on the asymptotic rate of growth and the asymptotically stable age distribution vector of the Leslie population model. This population model can be represented by a nonnegative matrix whose Perron root and, an appropriately normalized, Perron eigenvector then furnish the rate of growth and the stable age distribution of the model, respectively. The first and second order effects are obtained by computing the first and second order partial derivatives of the Perron root and Perron vector with respect to those matrix entries which represent the fecundity and survival rates of each age group. The existence of these derivatives is assured because in the problem's setting the Perron root is simple. It should be mentioned that first order effects of changes in the fecundity and survival rates upon the growth rates of the model have already been obtained by authors such as Demetrius [6], Goodman [12], and Lal and Anderson [15].

In the Leslie model one assumes that the population consists of $n$ age groups. Let $x_{i}(t)$ denote the number of individuals in the $i$-th age group at time $t$. Let $F_{i}, i=1, \ldots, n$, denote the fecundity of each individual in the $i$-th age group and let $P_{i}, i=1, \ldots, n-1$ denote the probability of survival of an individual from age $i$ to age $i+1$. Assume that both the fecundity and survival rates are independent of the time $t$. Then, as can be readily ascertained, the age distribution at time $t+1, t \geq 0$, can be described by the matrix - vector relation:

$$
x(t+1)=\left(\begin{array}{cccccc}
F_{1} & F_{2} & \ldots & \ldots & F_{n-1} & F_{n} \\
P_{1} & 0 & \ldots & \ldots & 0 & 0 \\
\vdots & \ddots & \vdots & \vdots & 0 & 0 \\
\vdots & \ldots & \ddots & \vdots & \vdots & \vdots \\
\vdots & \ldots & \ldots & \ddots & \vdots & \vdots \\
0 & \ldots & \ldots & \ldots & P_{n-1} & 0
\end{array}\right) x(t)=: \tilde{A} x(t),
$$

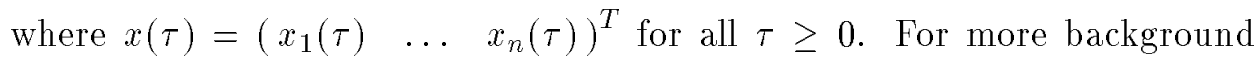
material on the Leslie population model see Pollard [18].

Papers in demography, cf. Demetrius [6], say that the population has reached a stationary age distribution if there exists a time $t_{0}$ and a constant 
$\lambda>0$ such that

$$
x(t+1)=\lambda x(t), \quad \forall t>t_{0} .
$$

In this case $\lambda$ is called the growth rate of the population. The term stationary age distribution comes from the fact that if (1.2) holds, then from time $t_{0}+1$ onwards the ratio between the various age groups in the population is maintained. We note further that if (1.2) holds, then

$$
\lambda x(t)=x(t+1)=\tilde{A} x(t)=\ldots=\tilde{A}^{t+1} x(0), \forall t>t_{0} .
$$

Because a stationary age distribution is reached in finite time only when the initial age distribution vector $x(0)$ is a linear combination of a Perron vector and a generalized null vector of $\tilde{A}$, we prefer to think of Demetrius' notions of growth rate of the population and stationary age distribution as the asymptotic growth rate and the asymptotically stable age distribution vector, respectively.

If $\tilde{A}$ is an irreducible matrix then by the Perron-Frobenius theory (see Section 2 for preliminaries and Berman and Plemmons [2] for a comprehensive background), $\lambda$ must be the Perron root of $\tilde{A}$ and $x(t)$ is a corresponding right Perron (eigen-)vector and the theory guarantees the existence and differentiability of both. Further, if $\tilde{A}$ is primitve, namely, it is irreducible with a single eigenvalue of maximum modulus, then, e.g., the power method for computing dominant eigenvalues and corresponding normalized eigenvectors (see Stewart [19, p.340]) shows that the right-hand-side of (1.3) will always converge to a Perron vector of $\tilde{A}$ so that asymptotically (1.2) holds. We comment now that just as the entries of the nonnegative matrix $\tilde{A}$ have a physical interpretation, we see that the Perron root and vector and their derivatives also have a physical meaning for the model.

One can relax the assumption of irreducibility. Even then the special structure of the Leslie matrix $\tilde{A}$ implies that its Perron root remains simple, thus ensuring that the Perron root and vector are still differentiable. While the reducible case may lead to some interesting questions, we nevertheless restrict ourselves to the case of irreducible Leslie matrices, - i.e. we will always assume that $F_{n}>0$ - since this captures the largest part of the mathematical content and difficulty.

It is natural to ask how the growth rate and the stable age distribution vectors are effected as we change the fecundity and survival probabilities at 
each age group. Demetrius [6] has investigated one of these questions by looking at the derivatives of the Perron root with respect to the fecundities and the survival rates which he obtained via the characteristic equation for a Leslie matrix. His results can also be obtained from a more general theorem giving expressions of the partial derivatives of a simple eigenvalue of a matrix with respect to the matrix entries as follows: Let $B=\left(b_{i, j}\right)$ be an $n \times n$ real or complex matrix and let $\mu$ be a simple eigenvalue of $B$. Let $\xi$ and $\eta$ be right and left eigenvectors of $B$ corresponding to $\mu$ normalized such that $\eta^{T} \xi=1$. Then it is known, see for example Stewart [19, p.305, Exer. 1], that

$$
\frac{\partial \mu}{\partial_{i, j}}=\xi_{j} \eta_{i}, \quad \forall 1 \leq i, j \leq n,
$$

where $\partial \mu / \partial_{i, j}$ is the derivative of $\mu$ with respect to the $(i, j)$-th entry at $B .{ }^{1}$

Consider the matrix $C=\mu I-B$. Zero now is a simple eigenvalue of $C$ and therefore the group generalized inverse of $C, C^{\#}$, exists and as is known, $\xi \eta^{T}=I-C C^{\#}$. Thus we see that the group inverse of $C$ can be used to express first order partial derivatives of $\mu$ with respect to the matrix entries at $B$. Further, as is shown in Deutsch and Neumann [7], the second order partial derivatives of $\mu$ with respect to the $(i, j)$-th entry can also written in terms of $C^{\#}$. Specifically they showed that

$$
\frac{\partial^{2} \mu}{\partial_{i, j}^{2}}=2\left(I-C C^{\#}\right)_{j, i} C_{j, i}^{\#}, \quad \forall 1 \leq i, j \leq n .
$$

Assume that $B$ is an $n \times n$ nonnegative and irreducible matrix. Since any right and left Perron vectors of $B$ are positive, (1.4) readily confirms the well known fact that the Perron value is a strictly increasing function in any of the matrix entries. In a series of papers Cohen [3,4], and [5] established the fact that the Perron root is a convex function in the main diagonal of the matrix. His principal approach to proving this fact was probabilistic relying on evolution equations due to Kac. In [7] a matrix theoretic proof of this fact is presented. Moreover, from the formula (1.5) which was found in [7] we see that for any pair $(i, j)$, the convexity or concavity of the Perron root with respect to $(i, j)$-th entry is determined by the sign of the $(j, i)$-th entry of $C^{\#}=(\lambda I-\tilde{B})^{\#}$. Perturbation and convexity theory of the Perron root

\footnotetext{
${ }^{1}$ Section 2 for a more precise discussion of the partial derivatives of eigenvalues of matrix with respect to matrix entries.
} 
has been investigated by a number of authors. To list a few we mention Elsner [9], Friedland [11], Golub and Meyer [13], Haviv, Ritov, and Rothblum [14], and Meyer and Stewart [17].

Let us return to our Leslie matrix $\tilde{A}$ and assume that it is irreducible. In this paper we explicitly compute expressions for the first and second order derivatives of the Perron root and an appropriately normalized Perron vector of a Leslie matrix with respect to its entries in the first row and on its subdiagonal. We shall then interpret what meaning our result have for the population model which the matrix represents. Most of our results show that younger ages exert more influence on the behavior of the growth rate and the stable age distribution vector than older age groups do. Our experience is that it is much more difficult to analyze the effects of changes in survival rates on the population than it is to do so for changes in the fecundity rates.

The plan of this paper is as follows. In Section 2 we shall present further notation and preliminaries which are necessary for the work here. We shall obtain in this section an explicit formula for the group inverse of $\lambda I-\tilde{A}$. In Section 3 we shall derive formulas for the second order derivatives of the Perron value of a Leslie matrix with respect to its top row and subdiagonal. In doing so some results of Deutsch and Neumann in [8] on the first and second derivatives of the Perron vector will be extended. In Section 4 we shall do similarly for the partial derivatives of the Perron vector of $\tilde{A}$. In each of Sections 3 and 4 we shall explain what implications our results have for the population model both qualitatively and, where possible, also quantitatively. 


\section{NOTATION AND PRELIMINARIES}

In this paper we shall use the following notation:

$\mathbb{R}^{k}$ denotes the $k$-dimensional real space.

$\mathbb{R}^{k, k}$ denotes the space of all $k \times k$ real matrices.

$e \in \mathbb{R}^{k}$ denotes the $k$-dimensional vector whose entries are all 1's.

$\boldsymbol{e}_{i} \in R^{k}$ denotes the $k$-dimensional unit coordinate vector, $i=1, \ldots, n$.

$E_{i, j} \in \mathbb{R}^{k, k}$ is the matrix whose $(i, j)$-th entry is 1 and whose remaining entries are 0 .

The symbol $\sim$ will indicate algebraic expressions with the same sign.

Let $u=\left(\begin{array}{lll}u_{1} & \ldots & u_{n}\end{array}\right) \in \mathbb{R}^{k}$ and $v=\left(\begin{array}{lll}v_{1} & \ldots & v_{n}\end{array}\right) \in \mathbb{R}^{k}$. We shall write that that $u \geq v$, if $u_{i} \geq v_{i}, i=1, \ldots, n$. We shall say that $u$ majorizes $v$, in notation $u \succeq v$, if

$$
\sum_{j=1}^{i} u_{j} \geq \sum_{j=1}^{i} v_{j}, \quad i=1, \ldots, n-1,
$$

and

$$
\sum_{j=1}^{n} u_{j}=\sum_{j=1}^{n} v_{j} .
$$

We shall say that a function $f: \mathbb{R}^{k} \rightarrow \mathbb{R}^{k}$ is isotone if

$$
u \succeq v \Rightarrow f(u) \succeq f(v) .
$$

Let $C \in \mathbb{R}^{n, n}$ and consider the matrix equations

$$
C X C=C, X C X=X \text {, and } C X=X C .
$$

A matrix $X \in \mathbb{R}^{n, n}$ which satisfies all three equations, if it exists, is called the group inverse of $C$ and is denoted by $C^{\#}$. Moreover, if $C^{\#}$ exists and $D$ is any nonsingular matrix, then

$$
\left(D^{-1} C D\right)^{\#}=D^{-1} C^{\#} D \text {. }
$$


In particular, if $D$ is a diagonal matrix whose diagonal entries are all positive, then all the entries of the matrices $C^{\#}$ and $D^{-1} C^{\#} D$ have identical signs in the same locations. It is known, see Ben-Israel and Greville [1], that a necessary and sufficient condition for $C^{\#}$ to exist is that the elementary divisors, if any, of $C$ corresponding to the eigenvalue zero are all linear. Thus if $B$ is an $n \times n$ nonnegative and irreducible matrix so that its Perron root is simple, then 0 is a simple eigenvalue of $C=\lambda I-B$, showing that the group inverse of $C$ exists.

Suppose that $B \in \mathbb{R}^{n, n}$ has a simple eigenvalue, call it $\mu(B)$. It readily follows from considerations involving the minimal polynomial that there is an open ball in $\mathbb{R}^{n, n}$ about $B$ such that every matrix in the ball has a simple eigenvalue. Thus for any $E \in \mathbb{R}^{n, n}$ and for sufficiently small $t \in \mathbb{R}, B+t E$ has a simple eigenvalue $\mu(B+t E)$ such that $\mu(B+t E) \rightarrow 0$ as $t \rightarrow 0$. Wilkinson [20, pp.66-67] shows that for sufficiently small $t, \mu(B+t E)$ can be expanded in a convergent power series about $B$. Hence the derivatives of all orders of $\mu$ with respect to $t$ exist at $B$. In particular, the partial derivative of $\mu$ with respect to the $(i, j)$-th entry at $B$ is given by the limit

$$
\frac{\partial \mu(B)}{\partial_{i, j}}:=\lim _{t \rightarrow 0} \frac{\mu\left(B+t E_{i, j}\right)-\mu(B)}{t} \underset{\text { by }}{\overline{(1.4)}} \xi_{j} \eta_{i},
$$

where $\xi=\xi(B)=\left(\begin{array}{lll}\xi_{1} & \ldots & \xi_{n}\end{array}\right)^{T}$ and $\eta=\eta(B)=\left(\begin{array}{lll}\eta_{1} & \ldots & \eta_{n}\end{array}\right)^{T}$ are right and left eigenvectors of $B$ corresponding to $\mu(B)$ normalized so that their inner product is 1 . In a similar manner we define the higher order partial derivatives of $\mu$ at $B$ with respect to the matrix entries. Wilkinson goes on to show that provided one of many standard normalizations (but not the infinity norm) is applied to the eigenvector corresponding to $\mu(B+t E)$ throughout the ball, then the entries of the corresponding eigenvector can be expanded in a convergent power series and therefore they too are differentiable with respect to $t$ at $B$. When it is absolutely clear from the context, we shall suppress the letter representing the matrix from the expressions for the partial derivatives, viz., we shall write $\partial \mu / \partial_{i, j}$ for $\partial \mu(B) / \partial_{i, j}$ etc.

Our approach will be to first develop our results for stochastic Leslie matrices and then transform them to general Leslie matrices from which we shall be able to draw our conclusion for the population model under consideration. To do so it will be helpful to have formulas connecting the partial derivatives with respect to the Perron root of a general nonnegative 
and irreducible matrix $\tilde{B}$ and the stochastic and irreducible matrix $B$ to which $\tilde{B}$ is transformed using the diagonal similarity

$$
B=\frac{1}{\lambda} D^{-1} \tilde{B} D
$$

where

$$
D=\left(\begin{array}{cccc}
\tilde{x}_{1} & 0 & \ldots & 0 \\
0 & \tilde{x}_{2} & \ldots & 0 \\
\vdots & \ddots & \ddots & \vdots \\
0 & \ldots & 0 & \tilde{x}_{n}
\end{array}\right)
$$

and where $\tilde{x}=\left(\tilde{x}_{1} \ldots \tilde{x}_{n}\right)^{T}$ is a right eigenvector of $\tilde{B}$ corresponding to its Perron root $\lambda$. Throughout we shall normalize all right Perron vectors so that their first entry equals 1 . Thus if $\tilde{y}$ is the left Perron vector of $\tilde{B}$ normalized so that $\tilde{x}^{T} \tilde{y}=1$, then it follows from (1.4), (1.5), (2.1), (2.3), and (2.4) that

$$
\frac{\partial^{2} \lambda}{\partial_{i, j}^{2}}=\frac{2 \tilde{y}_{i}}{\lambda} \frac{\left(\tilde{x}_{j}\right)^{2}}{\tilde{x}_{i}} Q_{j, i}^{\#},
$$

where $Q=I-B$. Similarly relations for the eigenvectors will also be useful. In particular, the following formulas can be obtained:

$$
\frac{\partial \tilde{x}}{\partial_{i, j}}=\frac{\tilde{x}_{j}}{\lambda \tilde{x}_{i}} D \frac{\partial x}{\partial_{i, j}}, \quad 1 \leq i, j \leq n,
$$

and

$$
\frac{\partial^{2} \tilde{x}}{\partial_{1, i}^{2}}=\frac{\tilde{y}_{1} \tilde{x}_{i}}{\lambda^{2}} D \frac{\partial^{2} x}{\partial_{1, i}^{2}}, \quad 1 \leq i \leq n .
$$

For the specific case of the Leslie matrix $\tilde{A}$ as given in (1.1), the right Perron vector is given by

$$
\tilde{x}=\left(\begin{array}{c}
1 \\
\frac{P_{1}}{\lambda} \\
\frac{P_{1} P_{2}}{\lambda^{2}} \\
\vdots \\
\frac{P_{1} \cdots P_{n-1}}{\lambda^{n-1}}
\end{array}\right) .
$$


With (2.3) and (2.4) in mind we find that $\tilde{A}$ can be transformed into the stochastic and irreducible Leslie matrix

$$
A=\left(\begin{array}{cccccc}
a_{1} & a_{2} & \ldots & \ldots & a_{n-1} & a_{n} \\
1 & 0 & \ldots & \ldots & 0 & 0 \\
\vdots & \ddots & \ddots & \vdots & 0 & 0 \\
\vdots & \ldots & \ddots & \ddots & \vdots & \vdots \\
\vdots & \ldots & \ldots & \ddots & \ddots & \vdots \\
0 & \ldots & \ldots & \ldots & 1 & 0
\end{array}\right)
$$

where

$$
a_{1}=\frac{F_{1}}{\lambda} \geq 0 \text { and } a_{i}=\frac{P_{1} \cdots P_{i-1} F_{i}}{\lambda^{i}} \geq 0, \quad i=2, \ldots, n,
$$

with $a_{n}>0$.

LEMMA 2.1 Let $A$ be a an irreducible stochastic Leslie matrix whose top row is given by $\left(\begin{array}{lll}a_{1} & \ldots & a_{n}\end{array}\right)$. Set

$$
Q=\left(\begin{array}{cccccc}
1-a_{1} & -a_{2} & \ldots & \ldots & -a_{n-1} & -a_{n} \\
-1 & 1 & \ldots & \ldots & 0 & 0 \\
\vdots & \ddots & \ddots & \vdots & 0 & 0 \\
\vdots & \ldots & \ddots & \ddots & \vdots & \vdots \\
\vdots & \ldots & \ldots & \ddots & \ddots & \vdots \\
0 & \ldots & \ldots & \ldots & -1 & 1
\end{array}\right)
$$

Then

$$
Q^{\#}=\left(\begin{array}{ll}
\left(Q^{\#}\right)_{1,1} & \left(Q^{\#}\right)_{1,2} \\
\left(Q^{\#}\right)_{2,1} & \left(Q^{\#}\right)_{2,2}
\end{array}\right)
$$

where

$$
\begin{gathered}
\left(Q^{\#}\right)_{1,1}=M^{-1}+\chi M^{-1} e r^{T} M^{-1}+\chi e r^{T} M^{-2}+\chi^{2}\left(r^{T} M^{-2} e\right) e r^{T} M^{-1} \\
\left(Q^{\#}\right)_{1,2}=-\chi M^{-1} e-\chi^{2}\left(r^{T} M^{-2} e\right) e \\
\left(Q^{\#}\right)_{2,1}=\chi r^{T} M^{-2}+\chi^{2}\left(r^{T} M^{-2} e\right) r^{T} M^{-1}
\end{gathered}
$$

and

$$
\left(Q^{\#}\right)_{2,2}=-\chi^{2}\left(r^{T} M^{-2} e\right)
$$


and where $e \in \mathbb{R}^{n-1}$,

$$
r=\left(\begin{array}{c}
0 \\
\vdots \\
-1
\end{array}\right) \in \mathbb{R}^{n-1} \text { and } \chi=\frac{1}{1-r^{T} M^{-1} e}=\frac{1}{\frac{1}{a_{n}} \sum_{i=0}^{n-1}\left(1-s_{i}\right)}
$$

and

$$
M^{-1}=\frac{1}{a_{n}}\left(\begin{array}{cccccc}
1-s_{0} & s_{n-1}-s_{1} & s_{n-1}-s_{2} & \ldots & s_{n-1}-s_{n-3} & s_{n-1}-s_{n-2} \\
1-s_{0} & 1-s_{1} & s_{n-1}-s_{2} & \ldots & s_{n-1}-s_{n-3} & s_{n-1}-s_{n-2} \\
1-s_{0} & 1-s_{1} & 1-s_{2} & \ldots & s_{n-1}-s_{n-3} & s_{n-1}-s_{n-2} \\
\vdots & \vdots & \vdots & \ddots & & \vdots \\
\vdots & \vdots & \vdots & & \ddots & \vdots \\
1-s_{0} & 1-s_{1} & 1-s_{2} & \ldots & 1-s_{n-3} & 1-s_{n-2}
\end{array}\right) .
$$

Here

$$
s_{0}=0 \text { and } s_{i}=\sum_{j=1}^{i} a_{j}, \quad \forall 1 \leq i \leq n-1 .
$$

Proof: Let $M$ be the $(n-1) \times(n-1)$ leading principal submatrix of $Q$. It is not difficult to check using the fact that the $a_{i}$ 's sum to 1 that $Q$ admits the full rank factorization

$$
Q=\left(\begin{array}{c}
M \\
r^{T}
\end{array}\right)\left(\begin{array}{ll}
I & -e
\end{array}\right)=: B C .
$$

According to Ben-Israel and Greville [1], $Q^{\#}=B(C B)^{-2} C$. Now it can be verified that $(C B)^{-1}=M^{-1}+\chi M^{-1} e r^{T} M^{-1}$, where $\chi$ and $M^{-1}$ are as given in (2.17) and (2.18), respectively. That $Q^{\#}$ is given by (2.12) and as specified in (2.13)-(2.19) can now be ascertained from the above partitioning of $C$ and $B$ and the aforementioned formula for $(C B)^{-1}$.

We comment that the above lemma is a specialization to the case of a singular M-matrix obtained from an $n \times n$ irreducible Leslie matrix of a formula for the group inverse of an a singular M-matrix obtained from a general $n \times n$ nonnegative and irreducible matrix found by Meyer [16, Theorem 5.2]. 


\section{THE PERRON ROOT OF A LESLIE MATRIX AS A FUNCTION OF TOP ROW AND SUBDIAGONAL}

We begin by determining the second order behavior of the Perron root as a function of the top row.

THEOREM 3.1 Let $A$ be an $n \times n$ irreducible stochastic Leslie matrix whose top row is given by $\left(\begin{array}{lll}a_{1} & \ldots & a_{n}\end{array}\right)$. Then the entries in the first column of the group inverse of $Q=I-A$ are given by

$$
Q_{i, 1}^{\#}=\frac{\chi}{a_{n}}\left[n-i-\frac{\chi}{a_{n}} \sum_{j=0}^{n-2}\left(1-s_{j}\right)(n-1-j)\right], \quad i=1, \ldots, n,
$$

where $\chi$ and the $s_{j}$ 's are as in (2.17) and (2.19). In particular, the entries of the first column of $Q^{\#}$ are strictly decreasing from first to last and $Q_{1,1}^{\#}>\left|Q_{n, 1}^{\#}\right|$. Moreover there exists an integer $k_{0}<(n+1) / 2$ such that $Q_{1,1}^{\#}, \ldots, Q_{k_{0}, 1}^{\#}$ are all nonnegative while $Q_{k_{0}+1,1}^{\#}, \ldots, Q_{n, 1}^{\#}$ are all nonpositive.

Proof: For each $1 \leq i \leq n-1$ we find from (2.13) that

$$
Q_{i, 1}^{\#}=e_{i}^{T}\left[M^{-1}+\chi M^{-1} e r^{T} M^{-1}+\chi e r^{T} M^{-2}+\chi^{2}\left(r^{T} M^{-2} e\right) e r^{T} M^{-1}\right] e_{1} .
$$

Also from (2.18) we have that

$$
r^{T} M^{-2} e=\left(r^{T} M^{-1}\right)\left(M^{-1} e\right)=\sum_{j=0}^{n-2} \frac{1-s_{j}}{a_{n}}(n-2-j)-\left(\sum_{j=0}^{n-2} \frac{1-s_{j}}{a_{n}}\right)^{2} .
$$

It now follows after several algebraic reductions that

$$
Q_{i, 1}^{\#}=\frac{\chi}{a_{n}}\left(1-s_{0}\right)\left(1-\chi r^{T} M^{-2} e-e_{i}^{T} M^{-1} e\right) .
$$

Further algebraic manipulations now yield (3.1). A similar calculation shows that (3.1) holds also for the case $i=n$.

From (3.1) it readily follows that the entries in the first column of $Q^{\#}$ are strictly decreasing from first to last and that the first entry is always positive 
(which is a general result of Meyer [16] for all diagonal entries of the group inverse of a singular and irreducible M-matrix and it is also an outcome of Cohen's results described in the introduction) while the last entry is always negative. Next, that $Q_{1,1}^{\#}>\left|Q_{n, 1}^{\#}\right|$ follows at once from (3.1). To complete the proof we need only show that if $i \geq(n+1) / 2$, then $Q_{i, 1}^{\#} \leq 0$. From (3.1) we find that for $2 \leq i \leq n-1, Q_{i, 1}^{\#}$ is nonpositive if and only if

$$
(n-i) \sum_{j=0}^{n-1}\left(1-s_{j}\right) \leq \sum_{j=0}^{n-1}\left(1-s_{j}\right)(n-1-j) .
$$

But (3.3) holds if and only if

$$
\sum_{j=0}^{n-1-i}\left(1-s_{j+i}\right)(j+1) \leq \sum_{j=0}^{i-2}\left(1-s_{j+2-i}\right)(j+1) .
$$

Since $1-s_{j+i} \leq 1-s_{j+2-i}$ for each $0 \leq j \leq n-i-1$, it now follows that if $i \geq(n+1) / 2$, then (3.4) always holds. Consequently, $Q_{i, 1}^{\#} \leq 0$ whenever $i \geq(n+1) / 2$.

Several comments are in order. Theorem 1 shows that the sign change in the first column of $Q^{\#}$ always occurs somewhere before the $\lfloor(n+1) / 2\rfloor$-th position. We first furnish an example to show that the sign change can occur at any position after the first and prior to the $(n+1) / 2-$ th if $n$ is odd or prior the $\lfloor(n+1) / 2\rfloor$ th if $n$ is even. Fix $2 \leq k<(n+1) / 2$. Let $A$ be given by (2.9) with

$$
a_{j}=\left\{\begin{array}{ll}
\alpha & \text { if } j=k-1 \\
1-\alpha & \text { if } j=n \\
0 & \text { otherwise }
\end{array},\right.
$$

where $0<\alpha<1$ is yet to be specified. From (3.1) we find that

$$
\begin{aligned}
Q_{k, 1}^{\#} & \sim n-k-\frac{\sum_{j=0}^{n-1}\left(1-s_{j}\right)(n-1-j)}{\sum_{j=0}^{n-1}\left(1-s_{j}\right)} \\
& \sim-\left[\frac{k(k-1)}{2}\right]+(1-\alpha)\left[\frac{(n-k)(n-k+1)}{2}\right] .
\end{aligned}
$$

Similarly,

$$
\begin{aligned}
Q_{k+1,1}^{\#} & \sim n-k-1-\frac{\sum_{j=0}^{n-1}\left(1-s_{j}\right)(n-1-j)}{\sum_{j=0}^{n-1}\left(1-s_{j}\right)} \\
& \sim-\left[\frac{k(k+1)}{2}\right]+\alpha+(1-\alpha)\left[\frac{(n-k)(n-k-1)}{2}\right] .
\end{aligned}
$$


Now choose a positive $\alpha$ so that

$$
\frac{n(n-1-2 k)}{(n-k)(n-k-1)-2}<\alpha<\frac{n(n+1-2 k)}{(n-k)(n-k+1)}<1 .
$$

Then a straightforward exercise shows that $Q_{k, 1}^{\#}>0>Q_{k+1,1}^{\#}$ and that such an $\alpha$ causes the sign change to occur at the desired admissible position.

Our next comment is motivated by the fact that if $\left(a_{1} \ldots a_{n}\right) \succeq$ $\left(\begin{array}{lll}\hat{a}_{1} & \ldots & \hat{a}_{n}\end{array}\right)$, then $\left(\begin{array}{lll}s_{1} & \ldots & s_{n}\end{array}\right) \succeq\left(\begin{array}{lll}\hat{s}_{1} & \ldots & \hat{s}_{n}\end{array}\right)$. Consider the function

$$
f(A)=f\left(a_{1}, \ldots, a_{n}\right)=\left(\begin{array}{lll}
Q_{1,1}^{\#} & \ldots & Q_{n, 1}^{\#}
\end{array}\right),
$$

where the $a_{i}$ 's, $1 \leq i \leq n$, are nonnegative and sum to 1 . Then is $f$ an isotone function? That in general the answer to this question is in the negative is illustrated by the example

$$
A=\left(\begin{array}{ccc}
\alpha / 2 & \alpha / 2 & 1-\alpha \\
1 & 0 & 0 \\
0 & 1 & 0
\end{array}\right) \text { and } \hat{A}=\left(\begin{array}{ccc}
0 & \alpha & 1-\alpha \\
1 & 0 & 0 \\
0 & 1 & 0
\end{array}\right)
$$

Notice that the top row of $A$ majorizes the top row of $\hat{A}$ for each $0<\alpha<1$. However, as can be verified, $f(\alpha / 2, \alpha / 2,1-\alpha)$ does not majorize $f(0, \alpha, 1-\alpha)$ for all $0<\alpha<1$ since, in particular, for $0<\alpha<(27-\sqrt{153}) / 16,(I-A)_{1,1}^{\#}>$ $(I-\hat{A})_{1,1}^{\#}$, whereas when $(27-\sqrt{153}) / 16<\alpha<1,(I-A)_{1,1}^{\#}<(I-\hat{A})_{1,1}^{\#}$. The following corollary shows that under certain conditions on $A$ and $\hat{A}$, there is an entrywise relationship between $f(A)$ and $f(\hat{A})$ :

COROLLARY 3.2 Let $a=\left(\begin{array}{lll}a_{1} & \ldots & a_{n}\end{array}\right)$ and $\hat{a}=\left(\begin{array}{lll}\hat{a}_{1} & \ldots & \hat{a}_{n}\end{array}\right)$ be the top rows of the irreducible stochastic Leslie matrices $A$ and $\hat{A}$, respectively. Suppose $a \succeq \hat{a}$. Then

$$
Q_{1,1}^{\#} \geq(\hat{Q})_{1,1}^{\#} \Rightarrow Q_{i, 1}^{\#} \geq(\hat{Q})_{i, 1}^{\#}, \quad \forall 1 \leq i \leq n,
$$

so that also $f(A) \succeq f(\hat{A})$ and

$$
Q_{n, 1}^{\#} \leq(\hat{Q})_{n, 1}^{\#} \Rightarrow Q_{i, 1}^{\#} \leq(\hat{Q})_{i, 1}^{\#}, \quad \forall 1 \leq i \leq n,
$$

so that also $f(A) \preceq f(\hat{A})$. 
Proof: Since $a \succeq \hat{a}$ we have from (3.1) that

$$
Q_{i, 1}^{\#}-Q_{k, 1}^{\#}=\frac{k-i}{\sum_{j=0}^{n-1}\left(1-s_{j}\right)} \leq \frac{k-i}{\sum_{j=0}^{n-1}\left(1-\hat{s}_{j}\right)}=(\hat{Q})_{i, 1}^{\#}-(\hat{Q})_{k, 1}^{\#}
$$

for all $1 \leq i \leq k \leq n$. Thus

$$
Q_{i, 1}^{\#}-(\hat{Q})_{i, 1}^{\#} \leq Q_{k, 1}^{\#}-(\hat{Q})_{k, 1}^{\#}
$$

for all $1 \leq i \leq k \leq n$ from which the results follow.

Next we examine the behavior of the Perron root of a Leslie matrix as a function of its entries on subdiagonal. From (1.5) and the preceding discussion we know that it suffices to determine the signs of the superdiagonal entries of $Q^{\#}$. As we shall see, determining expressions to represent these is no more difficult than determining expressions for the entries of $Q^{\#}$ down its first column. But determining the signs of the entries on the superdiagonal, especially in the top first half, appears to be more difficult than determining the signs of $Q^{\#}$ down the first column.

THEOREM 3.3 Let $A$ be an $n \times n$ irreducible stochastic Leslie matrix whose top row is given by $\left(\begin{array}{lll}a_{1} & \ldots & a_{n}\end{array}\right)$. Then, providing we interpret any summation sign from a lower limit which exceeds an upper limit to be zero, the entries in the super diagonal positions of the group inverse of $Q=I-A$ are given by

$$
\begin{gathered}
Q_{k, k+1}^{\#}=\chi\left\{\left(\frac{1-s_{k}}{a_{n}}\right)\left[n-k-1+-\chi \sum_{j=0}^{n-1}\left(\frac{1-s_{j}}{a_{n}}\right)(n-2-j)\right]\right. \\
\left.-\sum_{j=k}^{n-1}\left(\frac{1-s_{j}}{a_{n}}\right)\right\}, \quad k=1, \ldots, n-1,
\end{gathered}
$$

where $\chi$ and the $s_{j}$ 's are given by (2.17) and (2.19), respectively. The positive entries on the super diagonal of $Q^{\#}$, if any, appear consecutively from the $(1,2)$-th entry and form a non increasing sequence. The $(k, k+1)$-th entries, where $k \geq(n-1) / 2$, are all negative and for $k \geq(n+1) / 2$ those entries form a nondecreasing sequence.

Proof: For each $1 \leq k \leq n-2$, we find from (2.13) that

$Q_{k, k+1}^{\#}=e_{k}^{T}\left[M^{-1}+\chi M^{-1} e r^{T} M^{-1}+\chi e r^{T} M^{-2}+\chi^{2}\left(r^{T} M^{-2} e\right) e r^{T} M^{-1}\right] e_{k+1}$. 
Also recall the expression for $r^{T} M^{-2} e$ given in (3.2). Examining each term in the expansion of (3.7) and referring to (2.18) we find that

$$
\begin{gathered}
e_{k}^{T} M^{-1} e_{k+1}^{T}=\frac{1-s_{k}}{a_{n}}-1, \\
e_{k}^{T} M^{-1} e=\sum_{j=0}^{n-2} \frac{1-s_{j}}{a_{n}}-(n-k-1), \\
r^{T} M^{-1} e_{k+1}=-\frac{1-s_{k}}{a_{n}},
\end{gathered}
$$

and

$r^{T} M^{-2} e_{k+1}=\left(r^{T} M^{-1}\right)\left(M^{-1} e_{k+1}\right)=-\left[\frac{1-s_{k}}{a_{n}} \sum_{j=0}^{n-2} \frac{1-s_{j}}{a_{n}}-\sum_{j=0}^{k-1} \frac{1-s_{j}}{a_{n}}\right]$.

A number of algebraic reductions now yield (3.6). A similar calculation shows that (3.6) holds also when $k=n-1$.

To see that the positive entries in the super diagonal positions of $Q^{\#}$, if any, begin at the $(1,2)$ entry, are consecutive, and form a nonincreasing sequence we need only show that if $Q_{k, k+1}^{\#} \geq 0$, then $Q_{k-1, k}^{\#} \geq Q_{k, k+1}^{\#}$. To this end note that if $Q_{k, k+1}^{\#}>0$, then, in particular, from (3.6) it follows that

$$
n-k-1-\chi \sum_{j=0}^{n-1} \frac{1-s_{j}}{a_{n}}(n-2-j)>0 .
$$

But from (3.6) we find that

$$
Q_{k-1, k}^{\#}-Q_{k, k+1}^{\#}=\chi \frac{s_{k}-s_{k-1}}{a_{n}}\left[n-k-1-\chi \sum_{j=0}^{n-1} \frac{1-s_{j}}{a_{n}}(n-2-j)\right] .
$$

The claim now follows from (3.8).

Now let $k \geq(n-1) / 2$. From (3.6) we find that

$Q_{k, k+1}^{\#} \sim\left(1-s_{k}\right) \frac{\sum_{j=k}^{n-1}\left(1-s_{j}\right)(j-k)-\sum_{j=0}^{k-1}\left(1-s_{j}\right)(k-j)}{\sum_{j=0}^{n-1}\left(1-s_{j}\right)}-\sum_{j=k+1}^{n-1}\left(1-s_{j}\right)$. 
The numerator in the first term of the above expression can be rearranged as

$$
\sum_{j=1}^{n-k-1} j\left(s_{k-j}-s_{k+j}\right)-\sum_{j=n-k}^{k} j\left(1-s_{k-j}\right)
$$

which is evidently nonpositive. Consequently $Q_{k, k+1}^{\#} \leq 0$. Next consider the difference (3.9). Note that if $Q_{k, k+1}^{\#}$ is nonpositive and if

$$
n-k-1-\chi \sum_{j=0}^{n-1} \frac{1-s_{j}}{a_{n}}(n-2-j) \leq 0,
$$

then, necessarily, $Q_{k-1, k}^{\#} \leq Q_{k, k+1}^{\#} \leq 0$. But (3.10) holds if and only if

$$
a_{n}(n-k)+\sum_{j=k}^{n-2}\left(1-s_{j}\right)[j-(k-1)] \leq \sum_{j=0}^{k-1}\left(1-s_{j}\right)[(k-1)-j] .
$$

If $k \geq(n+1) / 2$, the terms in (3.11) can be paired-off to yield the equivalent expression:

$$
\begin{array}{r}
{\left[(k-1)-a_{n}(n-k)\right]} \\
+\left(s_{k}-s_{k-2}\right) \cdot 1 \\
+\left(s_{k+1}-s_{k-3}\right) \cdot 2 \\
+\ldots \\
+\left(s_{n-2}-s_{2 k-n}\right)(n-1-k) \\
+\left(1-s_{2 k-(n+1)}\right)(n-k) \\
+\ldots \\
+\left(1-s_{1}\right)(k-2) \quad \geq 0 .
\end{array}
$$

Observe that each of the terms on the left-hand-side above is nonnegative. Since we already proved that $Q_{n-1, n}^{\#} \leq 0$, the proof of the theorem is now complete.

Note that Theorem 3.3 implies that either the $Q_{k, k+1}^{\#}$ 's are all nonpositive or there is an index $k_{0}$, necessarily less than $(n-1) / 2$, such that for $1 \leq k \leq$ $k_{0}, Q_{k, k+1}^{\#} \geq 0$ and for $k_{0}+1 \leq k \leq n-1, Q_{k, k+1}^{\#} \leq 0$. While we are not able to show that there exist cases where $k_{0}$ attains the value $\lfloor(n-1) / 2\rfloor$, the following example shows that some $Q_{k, k+1}^{\#}$ can be positive when $n$ is 
sufficiently large and when $k$ is not too large compared with $n$. Specifically consider the $n \times n$ stochastic Leslie matrix whose top row is given by

$$
a_{j}= \begin{cases}\alpha & \text { if } j=k+1 \\ 1-\alpha & \text { if } j=n \\ 0 & \text { otherwise }\end{cases}
$$

where $\alpha$ is yet to be specified. From (3.6) we see that

$$
Q_{k, k+1}^{\#} \sim\left(1-s_{k}\right)\left[\frac{\sum_{j=0}^{n-1}\left(1-s_{j}\right)(j+1-k)}{\sum_{j=0}^{n-1}\left(1-s_{j}\right)}\right]-\sum_{j=k}^{n-1}\left(1-s_{j}\right) .
$$

Since $s_{j}=0$ for $0 \leq j \leq k$ and $s_{j}=\alpha$ for $k+1 \leq j \leq n-1$, (3.13) implies after some analysis that

$Q_{k, k+1}^{\#} \sim-k(k+1)+(1-\alpha)\left(n^{2}-4 k n+3 k^{2}+5 k-3 n+2\right)-2(1-\alpha)^{2}(n-k-1)^{2}$.

Choosing the value $\alpha=17 / 20$ and substituting it into (3.14), it follows that $Q_{k, k+1}^{\#}$ is positive as long as

$$
-119 k^{2}-(102 n+68) k+21 n^{2}-72 n+51>0 .
$$

Considering this as a quadratic in $k$, we find that $Q_{k, k+1}^{\#}$ is positive provided that

$$
k<\frac{-(102 n+68)+\sqrt{(102 n+68)^{2}+4(119)\left(21 n^{2}-72 n+51\right)}}{238} .
$$

For $k=1$, (3.15) yields that the minimal value of $n$ which guarantees that for the matrix given by (3.12), $Q_{1,2}^{\#}>0$, is $n=11$. For $k=2$, the minimal such $n$ is 17 . As $n \rightarrow \infty$, the right-hand-side (3.16) is asymptotic to

$$
n\left(\frac{-102+\sqrt{20400}}{238}\right) \approx 0.1715 n \text {. }
$$

Let us now revert to a general Leslie matrix $\tilde{A}$. From the discussion in Section 2 we see by (2.5) that the derivative of the Perron root with respect to the entries in the top row at $\tilde{A}$ is given by

$$
\frac{\partial^{2} \lambda}{\partial_{1, i}^{2}}=\frac{2}{\lambda}\left[\frac{1}{\sum_{j=0}^{n-1}\left(1-s_{j}\right)}\right]\left(\frac{P_{1} P_{2} \cdots P_{i-1}}{\lambda^{i-1}}\right)^{2} Q_{i, 1}^{\#}, \quad i=1, \ldots, n,
$$


where $Q=I-A$ and $A$ is as in (2.9) and (2.10). From this formula and the results of Theorem 3.1 it follows that if $\lambda>\max _{1 \leq i \leq n-1} P_{i}$, then

$$
\frac{\partial^{2} \lambda}{\partial_{1, i}^{2}} \geq 0 \Rightarrow \frac{\partial^{2} \lambda}{\partial_{1, i}^{2}} \geq \frac{\partial^{2} \lambda}{\partial_{1, j}^{2}}, j>i .
$$

In particular, for $\lambda$ as above,

$$
\max _{1 \leq i \leq n} \frac{\partial^{2} \lambda}{\partial_{1, i}^{2}}=\frac{\partial^{2} \lambda}{\partial_{1,1}^{2}}
$$

Similarly with respect to the subdiagonal entries we have that

$$
\frac{\partial^{2} \lambda}{\partial_{k+1, k}^{2}}=\frac{2 \lambda}{P_{k}^{2}} \frac{1-s_{k}}{\sum_{j=0}^{n-1}\left(1-s_{j}\right)} Q_{k, k+1}^{\#}, \quad k=1, \ldots, n-1 .
$$

In particular, if $P_{j} \geq P_{i}$ and $j>i$, then

$$
\frac{\partial^{2} \lambda}{\partial_{i+1, i}^{2}} \geq 0 \Rightarrow \frac{\partial^{2} \lambda}{\partial_{i+1, i}^{2}} \geq \frac{\partial^{2} \lambda}{\partial_{j+1, j}^{2}}
$$

We wish now to discuss the implications of the results obtained in this section on the population model which the Leslie matrix represents. First the qualitative interpretations. Theorems 3.1 and 3.3 show that the asymptotic rate of increase can only be a convex function of the fecundity and survival rates of younger age groups and that it is a concave function of these rates in older age groups. This suggests that only changes in the vital rates for younger age groups can yield a sharp change in the rate of increase of the asymptotic growth rate of the population. In attempting to compare the effects of changes in fecundity rates to changes in survival rates, we note that Theorem 3.1 always guarantees that for some younger age groups the asymptotic growth rate is a convex function of the fecundity. This is not necessarily the case for the effects of changes in the survival rates. Indeed, in our experience, it is difficult to produce examples where the asymptotic growth rate is a convex function of the survival rate of even the first age group. In this sense, the example given after Theorem 3.3 is atypical. It requires a peculiar fecundity distribution and, as we observe, a large number of age groups in order for changes in survival rates to have a sharp effect on the asymptotic growth rate. 
Next we consider the quantitative implications of our results upon the population model. Recall that Demetrius' result [6, p.134, eq.(8)] asserts that if $\lambda>\max _{1 \leq i \leq n-1} P_{i}$, then

$$
\frac{\partial \lambda}{\partial_{1, i}}>\frac{\partial \lambda}{\partial_{1, j}}, j>i .
$$

Our result in (3.19) reinforces (3.23) by showing that not only are the first partial derivatives of the asymptotic growth rate with respect to the fecundities ordered, but so are the second partial derivatives, at least for younger age groups. As for the effect of changes in the survival rates. Here Demetrius' result $[6$, p.134, eq.(11)] is that

$$
P_{j} \geq P_{i} \text { and } j>i \Rightarrow \frac{\partial \lambda}{\partial_{i+1, i}} \geq \frac{\partial \lambda}{\partial_{j+1, j}}
$$

Once again our result in (3.22) reinforces (3.24). Earlier we noted that the condition $\partial^{2} \lambda / \partial_{i+1, i}^{2} \geq 0$ which appears in $(3.22)$ can only occur for younger age group if at all. However when this is the case, the hypothesis $P_{j}>P_{i}$ for $j>i$ seems reasonable when $i$ is small because the survival rate for newborns is likely to be lower than that for slightly more mature individuals. 


\section{THE PERRON VECTOR OF A LESLIE MATRIX AS A FUNCTION OF TOP ROW AND SUBDIAGONAL}

In this section we shall examine the derivatives of the Perron vector of a stochastic Leslie matrix with respect to the entries in the top row and subdiagonal of the matrix. We shall than infer implications concerning the asymptotically stable age distribution vector of the general Leslie population model.

Let $\tilde{A}$ be an $n \times n$ general irreducible Leslie matrix whose Perron root is $\lambda=\lambda(\tilde{A})$. Throughout its right Perron vector $x=x(\tilde{A})$ will be normalized so that its first entry is equal to 1 . Denote by $y=y(\tilde{A})$ the left Perron vector of $\tilde{A}$ normalized so that $y^{T} x=1$. Since now all the derivatives of the first entry of that Perron vector are zero, it will be convenient for us to work with the truncated form of $x=\left(\begin{array}{llll}1 & x_{2} & \ldots & x_{n}\end{array}\right)^{T}$, viz. $\bar{x}=\left(\begin{array}{lll}x_{2} & \ldots & x_{n}\end{array}\right)^{T}$. Before truncation all vectors which we shall work with in this section will be in $\mathbb{R}^{n}$ and a bar over them shall indicate their truncation to an $(n-1)$ -

dimensional vector by deleting their first entry. This notation is consistent with [8] and for Leslie matrices, some results in that paper will also be generalized here.

In the interest of convenience we shall derive a basic relation for the derivatives of the $\bar{x}$ which in essence are already contained in [8]. Put $\tilde{Q}=$ $\lambda I-\tilde{A}$. On differentiating the matrix-vector relation $\tilde{A} x=\lambda x$ with respect to the $(i, j)$-th entry we obtain, on recalling (1.4), that

$$
E_{i, j} x+\tilde{A} \frac{\partial x}{\partial_{i, j}}=x_{j} y_{i} x+\lambda \frac{\partial x}{\partial_{i, j}}
$$

or

$$
\frac{\partial \bar{x}}{\partial_{i, j}}=x_{j} N_{\tilde{Q}}^{-1}\left(\overline{\epsilon_{i}}-y_{i} \bar{x}\right)
$$

where $N_{\tilde{Q}}$ is the $(n-1) \times(n-1)$ trailing principal submatrix of $\tilde{Q}$.

For the special case of a stochastic Leslie matrix we obtain the following:

LEMMA 4.1 At the $n \times n$ irreducible stochastic Leslie matrix A whose top 
row is given by $\left(\begin{array}{lll}a_{1} & \ldots & a_{n}\end{array}\right)$,

$$
\frac{\partial \bar{x}}{\partial_{1, i}}=-\frac{1}{\sum_{j=0}^{n-1}\left(1-s_{j}\right)}\left(\begin{array}{c}
1 \\
2 \\
\vdots \\
n-1
\end{array}\right), \quad 1 \leq i \leq n,
$$

and

$$
\frac{\partial \bar{x}}{\partial_{k+1, k}}=\frac{1}{\sum_{j=0}^{n-1}\left(1-s_{j}\right)}\left(\begin{array}{c}
-\left(1-s_{k}\right) \\
-2\left(1-s_{k}\right) \\
\vdots \\
-(k-1)\left(1-s_{k}\right) \\
\sum_{j=0}^{n-1}\left(1-s_{j}\right)-k\left(1-s_{k}\right) \\
\vdots \\
\sum_{j=0}^{n-1}\left(1-s_{j}\right)-(n-1)\left(1-s_{k}\right)
\end{array}\right), 1 \leq k \leq n-1
$$

where the $s_{i}$ 's are given in (2.19).

Proof: For the stochastic Leslie matrix $A$ we have $x(A)=\left(\begin{array}{lll}1 & \ldots & 1\end{array}\right)^{T}$ and for $Q=I-A$,

$$
N_{Q}^{-1}=\left(\begin{array}{cccc}
1 & 0 & \ldots & 0 \\
1 & 1 & \ldots & 0 \\
\vdots & \vdots & \ddots & \vdots \\
1 & 1 & \ldots & 1
\end{array}\right)
$$

Furthermore, if $y=y(A)$ is the left Perron vector of $A$ normalized as above, then

$$
y^{T}=\frac{1}{\sum_{j=0}^{n-1}\left(1-s_{j}\right)}\left(\begin{array}{llll}
\left(1-s_{0}\right) & \left(1-s_{1}\right) & \ldots & \left(1-s_{n-1}\right)
\end{array}\right) .
$$

The expression for $\partial \bar{x} / \partial_{1, i}$ now follows on substituting these values of $x, y$ and $N_{Q}^{-1}$ into (4.1) and on noting that $\overline{\epsilon_{1}}=0$. The expression for $\partial \bar{x} / \partial_{k+1, k}$ follows similarly.

Considering (4.2) we see that each entry of $\partial \bar{x} / \partial_{1, i}$ is negative for each $i=1, \ldots, n$. This fact is actually a consequence of a more general result of Elsner, Johnson, and Neumann [10, Theorem 1]. In contrast to (4.2), (4.3) shows that the signs of the entries of the $\partial \bar{x} / \partial_{k+1, k}$ are not necessarily uniform. Clearly the first $k-1$ entries are negative and the remaining ones 
form a decreasing sequence. The following argument shows that $\partial \bar{x} / \partial_{k+1, k}$ always has at least one positive entry. Note that

$\sum_{j=0}^{n-1}\left(1-s_{j}\right)-(k+1)\left(1-s_{k}\right)=\sum_{j=0}^{k}\left(s_{k}-s_{j}\right)+\sum_{j=k+1}^{n-1}\left(1-s_{j}\right)>0,1 \leq k \leq n-2$.

Thus we see that the $k$-th and $(k+1)$-th entries of $\partial \bar{x} / \partial_{k+1, k}$ are always positive for $1 \leq k \leq n-2$. A similar argument shows that the last entry of $\partial \bar{x} / \partial_{n, n-1}$ is also always positive. From the above we see that either the entries of $\partial \bar{x} / \partial_{k+1, k}$ in positions $k$ through $n-1$ are all nonnegative or there is an index $i_{0} \geq k+1$ such that the entries in positions $k$ through $i_{0}$ are nonnegative and the remaining entries are nonpositive. To see that each such sign pattern can be realized consider the stochastic Leslie matrix whose top row has $0<\alpha<1$ in the $(k+1)$-th position and $1-\alpha$ in the $n-$ th position. It is readily verified that each admissible sign pattern can be obtained by a suitable choice of $\alpha$.

We next investigate the sign pattern of the second partial derivatives of $\bar{x}$ with respect to the entries of the top row of a stochastic Leslie matrix.

THEOREM 4.2 Let $A$ be an $n \times n$ irreducible stochastic Leslie matrix whose top row is given by $\left(\begin{array}{lll}a_{1} & \ldots & a_{n}\end{array}\right)$ and let $x=x(A)$ be its right Perron vector normalized so that its first entry is 1 . Then

$$
\left(\frac{\partial^{2} \bar{x}}{\partial_{1, i}^{2}}\right)_{l}=2 l\left(\frac{1}{\sum_{j=0}^{n-1}\left(1-s_{j}\right)}\right)^{2}\left[\frac{l+1}{2}+i-\frac{\sum_{j=0}^{n-1}\left(1-s_{j}\right)(j+1)}{\sum_{j=0}^{n-1}\left(1-s_{j}\right)}\right]
$$

for all $1 \leq l \leq n-1$ and $1 \leq i \leq n$ and where the $s_{i}$ 's are as specified in (2.19). Furthermore, when $i$ is fixed, there is at most one sign change in the entries of $\partial^{2} \bar{x} / \partial_{1, i}^{2}$ from minus to plus as l increases. Similarly, when $l$ is fixed and $i$ is increased, there is at most one sign change in the sequence formed from the $l$-th entries of the vectors $\partial^{2} \bar{x} / \partial_{1, i}^{2}, i=1, \ldots, n$. In particular,

$$
\frac{l+1}{2}+i \geq \frac{n+1}{2} \Rightarrow\left(\frac{\partial^{2} \bar{x}}{\partial_{1, i}^{2}}\right)_{l} \geq 0
$$

and an all pluses sign pattern is possible when either $i$ or $l$ is sufficiently large. 
Proof: As usual let $Q=I-A$ and let $y$ be the left Perron vector of $A$ normalized so that $y^{T} x=1$. From formula (4.13) in [8] we find that

$$
\frac{\partial^{2} \bar{x}}{\partial_{1, i}^{2}}=2 y_{1} x_{i}\left(y_{1} x_{i} N_{Q}^{-1}-Q_{i, 1}^{\#} I\right) N_{Q}^{-1} \bar{x}, \quad i=1, \ldots, n .
$$

Substituting in the expressions for $x, y$, and $N_{Q}^{-1}$ developed in Lemma 4.2 and using the formula for $Q_{i, 1}^{\#}$ given in (3.1) yields after some simplification (4.4). The claims concerning the sign patterns follows readily by inspecting (4.4). That (4.5) holds can be established by the same argument given in Theorem 3.1 which was used to show that $Q_{i, 1}^{\#} \leq 0$ for $i \geq(n+1) / 2$.

As in our remarks following Theorem 3.1 concerning the sign pattern of the entries of the first column of $Q^{\#}$, examples can be constructed to show that when $i$ is fixed and $l$ is increasing, the switch from minus to nonnegative in $\left(\partial^{2} \bar{x} / \partial_{1, i}^{2}\right)_{l}$ can occur at any index $l_{0}<n-2 i$. A similar remark holds for the sequence $\partial^{2} \bar{x} / \partial_{1, i}^{2}$ when $l$ is fixed and $i$ is increasing.

In the spirit of Corollary 3.2 we can prove the following majorization result for the second derivatives of the Perron vectors corresponding to two stochastic Leslie matrices:

COROLLARY 4.3 Let $a=\left(\begin{array}{lll}a_{1} & \ldots & a_{n}\end{array}\right)$ and $\hat{a}=\left(\begin{array}{lll}\hat{a}_{1} & \ldots & \hat{a}_{n}\end{array}\right)$ be the top rows of the irreducible stochastic Leslie matrices $A$ and $\hat{A}$, respectively. Suppose $a \succeq \hat{a}$. Then

$$
\frac{\partial^{2} \bar{x}}{\partial_{1,1}^{2}} \geq \frac{\partial^{2} \overline{\hat{x}}}{\partial_{1,1}^{2}} \Rightarrow \frac{\partial^{2} \bar{x}}{\partial_{1, i}^{2}} \geq \frac{\partial^{2} \overline{\hat{x}}}{\partial_{1, i}^{2}}, \quad 1 \leq i \leq n,
$$

and

$$
\frac{\partial^{2} \bar{x}}{\partial_{1, n}^{2}} \leq \frac{\partial^{2} \overline{\hat{x}}}{\partial_{1, n}^{2}} \Rightarrow \frac{\partial^{2} \bar{x}}{\partial_{1, i}^{2}} \leq \frac{\partial^{2} \overline{\hat{x}}}{\partial_{1, i}^{2}}, \quad 1 \leq i \leq n .
$$

Proof: From (4.4) we have that

$$
\frac{\partial^{2} \bar{x}}{\partial_{1, i}^{2}}-\frac{\partial^{2} \bar{x}}{\partial_{1, j}^{2}}=2(i-j)\left[\frac{1}{\sum_{j=0}^{n-1}\left(1-s_{j}\right)}\right]^{2}\left(\begin{array}{c}
1 \\
2 \\
\vdots \\
n-1
\end{array}\right)
$$




$$
\leq 2(i-j)\left[\frac{1}{\sum_{j=0}^{n-1}\left(1-\hat{s}_{j}\right)}\right]^{2}\left(\begin{array}{c}
1 \\
2 \\
\vdots \\
n-1
\end{array}\right)=\frac{\partial^{2} \overline{\hat{x}}}{\partial_{1, i}^{2}}-\frac{\partial^{2} \overline{\hat{x}}}{\partial_{1, j}^{2}}
$$

for $1 \leq j \leq i \leq n$ and where the $s_{i}$ 's are specified in (2.19) and where the $\hat{s}_{i}$ 's are similarly determined. Consequently

$$
\frac{\partial^{2} \bar{x}}{\partial_{1, i}^{2}}-\frac{\partial^{2} \overline{\hat{x}}}{\partial_{1, i}^{2}} \geq \frac{\partial^{2} \bar{x}}{\partial_{1, j}^{2}}-\frac{\partial^{2} \overline{\hat{x}}}{\partial_{1, j}^{2}}, \quad 1 \leq j \leq i \leq n,
$$

from which the results follow.

We now return to a general Leslie matrix $\tilde{A}$. From (2.6) we have that for the first partial derivatives of the Perron vector with respect to the top row and subdiagonal,

$$
\frac{\partial \overline{x(\tilde{A})}}{\partial_{1, i}}=\frac{P_{1} P_{2} \cdots P_{i-1}}{\lambda^{i}} N_{D} \frac{\partial \overline{x(A)}}{\partial_{1, i}}, \quad i=1, \ldots, n,
$$

and

$$
\frac{\partial \overline{x(\tilde{A})}}{\partial_{k+1, k}}=\frac{1}{P_{k}} N_{D} \frac{\partial \overline{x(A)}}{\partial_{k+1, k}}, \quad k=1, \ldots, n-1,
$$

where $A$ is given by (2.9) and (2.10). Similarly, for the second partial derivatives of the Perron vector with respect to the first row we have from (2.7) that

$$
\frac{\partial^{2} \overline{x(\tilde{A})}}{\partial_{1, i}^{2}}=\left[\frac{1}{\overline{\sum_{j=0}^{n-1}\left(1-s_{j}\right)}}\right]\left(\frac{P_{1} P_{2} \cdots P_{i-1}}{\lambda^{i}}\right)^{2} N_{D} \frac{\partial^{2} \overline{x(A)}}{\partial_{1, i}^{2}}
$$

for all $i=1, \ldots, n$. From (4.8) we can conclude that

$$
\lambda \geq \max _{1 \leq i \leq n-1} P_{i} \text { and }\left(\frac{\partial \overline{x(\tilde{A})}}{\partial_{k+1, k}}\right)_{j} \geq 0 \Rightarrow\left(\frac{\partial \overline{x(\tilde{A})}}{\partial_{k+1, k}}\right)_{j} \geq\left(\frac{\partial \overline{x(\tilde{A})}}{\partial_{k+1, k}}\right)_{l}, l \geq j
$$

In particular

$$
\max _{1 \leq j \leq n-1}\left(\frac{\partial \overline{x(\tilde{A})}}{\partial_{k+1, k}}\right)_{j}=\left(\frac{\partial \overline{x(\tilde{A})}}{\partial_{k+1, k}}\right)_{k}
$$


We come now to interpret our results on the Perron vector for the population model. We begin with qualitative observations. For our analysis of the behavior of the asymptotically stable age distribution vector to make sense we have to choose a frame of reference, and the one we select is to compare the sizes of any age group group to the size of the first age group.

(i) Formula (4.2) in Lemma 4.1 implies that raising the fecundity of any age group decreases the ratio of the size of any age group beyond the first to the size of the first age group.

(ii) Formula (4.3) in Lemma 4.1 shows that raising the survival rate of the $k$-th age group has the effect of increasing the ratio of the size of the $(k+1)$-th age group to the size of the first age group and possibly of raising those ratios for subsequent age groups while diminishing the those ratios for age groups $2, \ldots, k$.

In the way of quantitative interpretation of our results on the Perron vector for the population model, the only definite conclusion that we can draw follows from (4.3) and (4.11). Here we see that increasing the survival rate at the $k$-th age group has the effect of raising the ratios of the sizes of some age groups beyond the size of the first to the first age group while decreasing others. However, when $\lambda \geq \max _{1 \leq i \leq n-1} P_{i}$, it follows from (4.8) that the ratio which increases the most corresponds to the $(k+1)$-th age group.

ACKNOWLEDGEMENTS This research was conducted while the first author was a Postdoctoral Fellow at the Institute for Mathematics and its Applications of the University of Minnesota and while the second author was a visitor there. Both authors wish to thank the IMA and its staff for their support. 


\section{References}

[1] A. Ben-Israel and T. N. Greville. Generalized Inverses: Theory and Applications. Academic Press, New-York, 1973.

[2] A. Berman and R. J. Plemmons. Nonnegative Matrices in the Mathematical Sciences. Academic Press, New-York, 1979.

[3] J. E. Cohen. Derivatives of the spectral radius as a function of a nonnegative matrix. Math. Proc. Cambridge Philos. Soc., 83:183-190, 1978.

[4] J. E. Cohen. Random evolutions and the spectral radius of a nonnegative matrix elements. Math. Proc. Cambridge Philos. Soc., 86:343$350,1979$.

[5] J. E. Cohen. Convexity of the dominant eigenvalue of an essentially non-negative matrix. Proc. Amer. Math. Soc., 81:657-658, 1981.

[6] L. Demetrius. The sensitivity of population growth rate to perturbations in the life cycle components. Math. Biosciences, 4:129-136, 1969.

[7] E. Deutsch and M. Neumann Derivatives of the Perron root at an essentially nonnegative matrix and the group inverse of an M-matrix. J. Math. Anal. Appl., 102: 1-29, 1984.

[8] E. Deutsch and M. Neumann On the first and second order derivatives of the Perron vector. Lin. Alg. Appl., 71:57-76, 1985.

[9] L. Elsner. On convexity properties of the spectral radius of nonnegative matrices. Lin. Alg. Appl., 61:31-35, 1984.

[10] L. Elsner, C.R. Johnson, and M. Neumann. On the effect of the perturbation of a nonnegative matrix on its Perron eigenvector. Czech. Math. J., 32:99-109, 1982.

[11] S. Friedland. Convex spectral functions. Lin. Multilin. Alg, 9:299-316, 1981.

[12] L. A. Goodman. On the sensitivity of the intrinsic growth rate to changes in the age-specific birth and death rates. Theoretical Population Biology, 2:339-354, 1971. 
[13] G. H. Golub and C. D. Meyer, Jr. Using the QR-factorization and group inversion to compute, differentiate, and estimate the sensitivity of stationary probabilities for Markov chains. SIAM J. Alg. Discrete Methods, 7:273-281, 1986.

[14] M. Haviv, Y. Ritov, U. G. Rothblum. Taylor expansions of eigenvalues of perturbed matrices with applications to spectral radii of nonnegative matrices. Lin. Alg. Appl., 168:159-188, 1992.

[15] R. Lal and D. H. Anderson. Calculation and utilization of component matrices in linear bioscience models. Math. Biosciences, 99:11-29, 1990.

[16] C. D. Meyer, Jr. The role of the group generalized inverse in the theory of finite Markov chains. SIAM Rev., 17:443-464, 1975.

[17] C. D. Meyer, Jr. and G. W. Stewart. Derivatives and perturbations of eigenvectors. SIAM J. Numer. Anal., 25:679-691, 1988.

[18] J. H. Pollard. Mathematical Models for the Growth of Human Populations. Cambridge Univ. Press, Cambridge, 1973.

[19] G. W. Stewart. Introduction to Matrix Computations. Academic Press, New York, 1973.

[20] J. H. Wilkinson. The Algebraic Eigenvalue Problem. Oxford Univ. Press, London, 1965. 